OPEN ACCESS

Edited by: Kerstin Feistel,

University of Hohenheim, Germany

Reviewed by:

Mareike Albert,

Technical University Dresden,

Germany

Tran Tuoc,

Ruhr University Bochum, Germany

*Correspondence:

Silke Paull

silke.pauli@med.uni-goettingen.de

Annette Borchers

borchers@uni-marburg.de

Specialty section:

This article was submitted to

Morphogenesis and Patterning,

a section of the journal

Frontiers in Cell and Developmental

Biology

Received: 17 September 2021

Accepted: 22 October 2021

Published: 05 November 2021

Citation:

Pauli S, Berger $H$, Ufartes $R$ and Borchers A (2021) Comparing a Novel Malformation Syndrome Caused by

Pathogenic Variants in FBRSL1 to

AUTS2 Syndrome.

Front. Cell Dev. Biol. 9:779009.

doi: 10.3389/fcell.2021.779009

\section{Comparing a Novel Malformation Syndrome Caused by Pathogenic Variants in FBRSL1 to AUTS2 Syndrome}

\author{
Silke Pauli ${ }^{1 *}$, Hanna Berger ${ }^{2}$, Roser Ufartes ${ }^{1}$ and Annette Borchers ${ }^{2 *}$ \\ ${ }^{1}$ Institute of Human Genetics, University Medical Center Göttingen, Göttingen, Germany, ${ }^{2}$ Faculty of Biology, Molecular \\ Embryology, Philipps-University Marburg, Marburg, Germany
}

Truncating variants in specific exons of Fibrosin-like protein 1 (FBRSL1) were recently reported to cause a novel malformation and intellectual disability syndrome. The clinical spectrum includes microcephaly, facial dysmorphism, cleft palate, skin creases, skeletal anomalies and contractures, postnatal growth retardation, global developmental delay as well as respiratory problems, hearing impairment and heart defects. The function of FBRSL1 is largely unknown, but pathogenic variants in the FBRSL1 paralog Autism Susceptibility Candidate 2 (AUTS2) are causative for an intellectual disability syndrome with microcephaly (AUTS2 syndrome). Some patients with AUTS2 syndrome also show additional symptoms like heart defects and contractures overlapping with the phenotype presented by patients with FBRSL1 mutations. For AUTS2, a dual function, depending on different isoforms, was described and suggested for FBRSL1. Both, nuclear FBRSL1 and AUTS2 are components of the Polycomb subcomplexes PRC1.3 and PRC1.5. These complexes have essential roles in developmental processes, cellular differentiation and proliferation by regulating gene expression via histone modification. In addition, cytoplasmic AUTS2 controls neural development, neuronal migration and neurite extension by regulating the cytoskeleton. Here, we review recent data on FBRSL1 in respect to previously published data on AUTS2 to gain further insights into its molecular function, its role in development as well as its impact on human genetics.

Keywords: FBRSL1, AUTS2, malformation syndrome, embryonic development, polycomb complex

\section{FBRSL1 VARIANTS CAUSE A NOVEL DISABILITY SYNDROME WITH AN OVERLAPPING PHENOTYPE TO AUTS2 SYNDROME}

Recently, we identified truncating variants in the FBRSL1 gene in three unrelated children with an unknown malformation syndrome (Ufartes et al., 2020). The patients presented with respiratory insufficiency and feeding difficulties in the neonatal period. During infancy, intellectual disability, no active speech, postnatal microcephaly, growth retardation and contractures became apparent. In addition, two of the three patients showed cleft palate and heart defects (one with atrial septal defect and persistent ductus arteriosus, one with atrial septal defect and ventricular septal defect). In one patient asplenia and in another patient anal atresia were observed. Furthermore, the two more severely affected patients were born with pronounced congenital skin creases at the back, the arms, and legs. During the first year of life the skin creases became less pronounced and disappeared 
TABLE 1 | Comparison of clinical features seen in patients with FBRSL1 mutation and patients with AUTS2 syndrome.

Clinical findings

FBRSL1 syndromic phenotype

AUTS2 syndrome

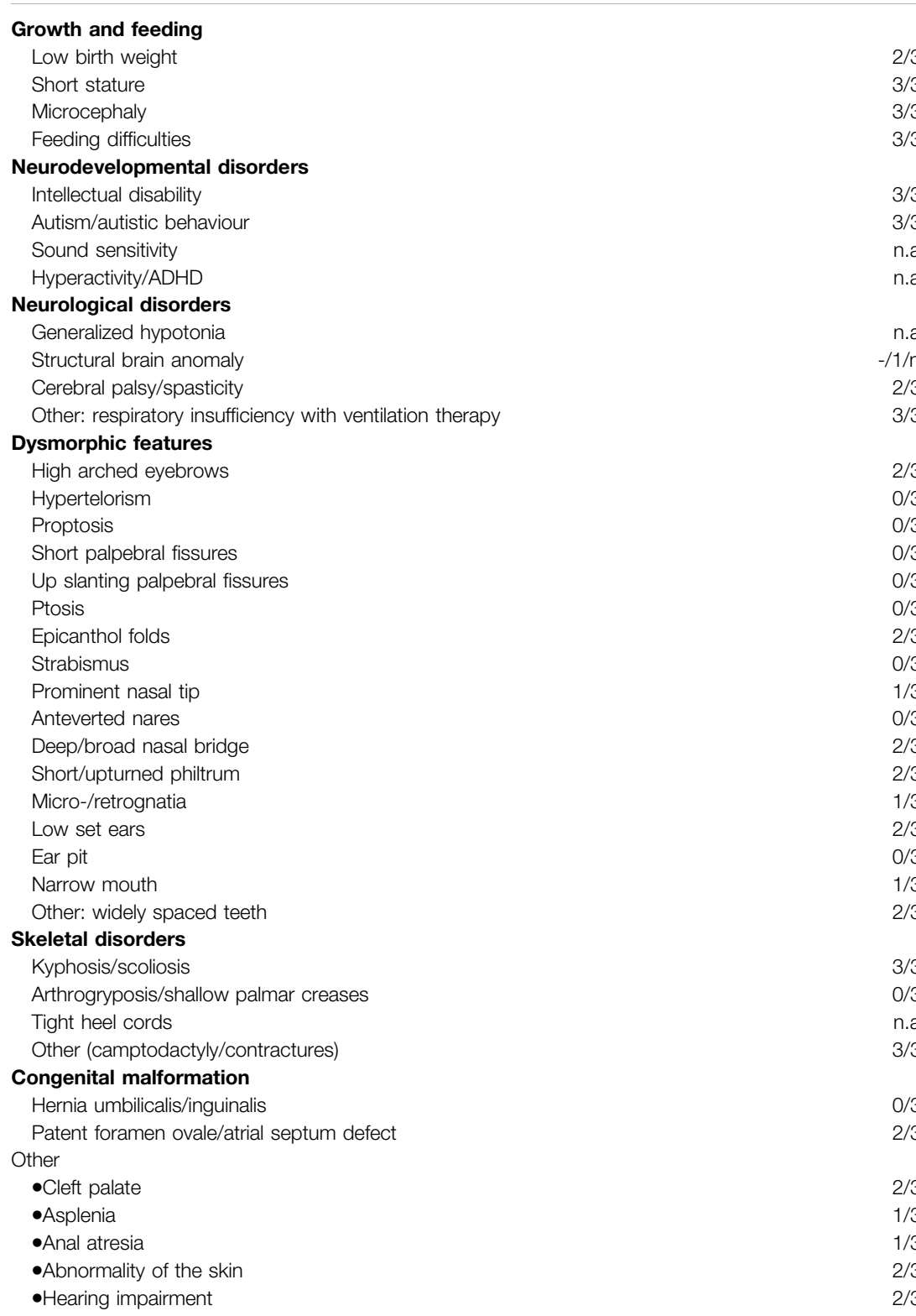

10/54 (18,5\%)

$26 / 59(44,1 \%)$

$37 / 57(64,9 \%)$

$33 / 55(60,0 \%)$

64/66 (97,0\%)

$16 / 40(40,0 \%)$

$28 / 56(50,0 \%)$

$17 / 28(60,7 \%)$

$23 / 60(38,3 \%)$

$11 / 46(23,9 \%)$

20/57 (35,1\%)

$13 / 37(35,1 \%)$

$14 / 37(37,8 \%)$

7/37 (18,9\%)

9/37 (24,3\%)

$5 / 37(13,5 \%)$

$11 / 37(29,7 \%)$

$8 / 37(21,6 \%)$

9/37 (24,3\%)

7/37 (18,9\%)

7/37 (18,9\%)

$12 / 37(32,4 \%)$

$11 / 37(29,7 \%)$

$11 / 36(30,6 \%)$

$11 / 36(30,6 \%)$

$5 / 36(13,9 \%)$

$16 / 37(43,2 \%)$

$10 / 47(21,3 \%)$

$6 / 28(21,4 \%)$

$6 / 13(46,2 \%)$

$6 / 59(10,2 \%)$

$4 / 26(15,4 \%)$

The clinical feature terminology is based on the list of features used for the AUTS2 syndrome severity scoring system (Beunders et al., 2013). The data for AUTS2 syndrome were adapted from Sanchez-Jimeno et al. which is based on nine different studies (Sultana et al., 2002; Kalscheuer et al., 2007; Bakkaloglu et al., 2008; Huang et al., 2010; Girirajan et al., 2011; Jolley et al., 2013; Nagamani et al., 2013; Liu et al., 2015; Beunders et al., 2016). In addition, the data include a cohort of five patients published by Sanchez-Jimeno et al. (Sanchez-Jimeno et al., 2021). The data for the FBRSL1 syndromic phenotype is based on the three patients published in Ufartes et al. (Ufartes et al., 2020). Abbreviations: $n . a .=n o t$ assessed, ADHD = attention deficit/hyperactivity disorder. A remarkable overlap between the two syndromes was observed with a wider spectrum and higher rate of congenital malformations in children with a pathogenic variant in FBRSL1.

(Ufartes et al., 2020). Interestingly, the clinical phenotype of the newly described malformation syndrome caused by FBRSL1 variants partially overlaps with the severe form of AUTS2 syndrome (Table $\mathbf{1}$ ).

AUTS2 syndrome (MIM 615834) was first described in 2013 (Beunders et al., 2013), as a neurodevelopmental disorder caused by pathogenic variants and deletions of the AUTS2 gene (MIM 607270 , activator of transcription and developmental regulator).
Depending on the location of AUTS2 point mutations/deletions the phenotype ranges from an isolated neurodevelopmental disorder (e.g., autism spectrum disorder, attention deficit hyperactivity disorder, learning disabilities and/or intellectual disability) to a syndromic disorder with microcephaly, short stature, feeding difficulties, heart defects, skeletal anomalies, contractures and dysmorphic features (Beunders et al., 2013; Beunders et al., 2016; Saeki et al., 2019; Sanchez-Jimeno et al., 
2021). To date, more than 60 patients with AUTS2 syndrome have been described in the literature and most of them carry an intragenic de novo deletion of AUTS2, whereas point mutations leading to the disease are rarely described (Sanchez-Jimeno et al., 2021). Due to a high inter- and intrafamilial variability an AUTS2 syndrome severity scoring system (ASSS) was established by Beunders and colleagues 2013. The scoring system is based on 32 features seen with a frequency of over $10 \%$ in AUTS2 syndrome patients of the first described cohort (Beunders et al., 2013). The ASSS revealed that patients with small deletions at the N-terminus of AUTS2 typically present a mild phenotype; in some cases, these deletions were inherited from a mildly or unaffected parent (Beunders et al., 2013). In contrast, deletions of the C-terminus of AUTS2 are mostly associated with a severe AUTS2 syndrome phenotype combining neurodevelopmental features with malformations and dysmorphic features (Beunders et al., 2013). Therefore, it was suggested that the AUTS2 C-terminus plays a critical role in AUTS2 syndrome (Beunders et al., 2013; Beunders et al., 2016; Saeki et al., 2019; Sanchez-Jimeno et al., 2021). Interestingly, the situation seems to be different for the truncating FBRSL1 variants characterized in Ufartes et al. (2020), which all localized to the $\mathrm{N}$-terminus of the FBRLS1 gene. As the patients carrying FBRSL1 variants showed features associated with AUTS2 syndrome, we also used the ASSS to compare the phenotype of the three patients with a variant in FBRSL1 (Ufartes et al., 2020) to patients with AUTS2 syndrome (Table 1). A remarkable clinical overlap between the FBRSL1 syndromic phenotype and the severe form of AUTS2 syndrome was observed. Although, so far only three patients with the FBRSL1 syndromic phenotype have been described (Ufartes et al., 2020), it seems that they show a wider range of congenital malformations compared to the symptoms observed in AUTS2 patients. To gain insight into common and distinct functions of FBRSL1 and AUTS2, we take a closer look at their evolutionary conservation and potential functions.

\section{FBRSL1 AND AUTS2 ARE PARALOGS THAT LIKELY SHARE CONSERVED FUNCTIONS}

FBRSL1 and AUTS2 belong to a tripartite gene family, the AUTS2 family, which also includes Fibrosin (FBRS) (Singh et al., 2015). The AUTS2 family is predicted to be an ohnolog gene family (Singh et al., 2015), representing a group of paralog genes generated by two rounds of whole genome duplication during vertebrate evolution and frequently implicated in human disease (Dickerson and Robertson, 2012; Singh et al., 2012; Malaguti et al., 2014; Mclysaght et al., 2014). The AUTS2 family ohnologs show a large overlap of conserved regions, but also unique elements which likely contribute to the functional diversity of the proteins (Sellers et al., 2020). Detailed information about the conserved regions shared by AUTS2-related proteins as well as an evolutionary analysis of the AUTS2 family can be found in Sellers et al., 2020 (Sellers et al., 2020). Based on their extended phylogenetic analysis, Sellers et al. recommended to rename FBRSL1 to AUTS2-like Protein 1, because AUTS2 and FBRSL1 share a most recent common ancestor, suggesting that these proteins are evolutionary closer related to each other than to FBRS (Sellers et al., 2020). Thus, it is intriguing to speculate that both proteins may share common functions, which may also explain their overlapping phenotypes observed in the respective syndromes.

Research using animal model systems indicate that FBRSL1 and AUTS2 share common functions in vertebrate development. As Auts2 function in neurodevelopmental disorders has been addressed in a number of comprehensive reviews (Oksenberg and Ahituv, 2013; Hori and Hoshino, 2017; Pang et al., 2021), we will here only briefly discuss its role in mouse and zebrafish development. In the mouse, Auts 2 is broadly expressed in the developing brain, with high expression in key areas of higher cognitive brain function (Bedogni et al., 2010). Heterozygous disruption of Auts2 results in similar symptoms as seen in AUTS2 syndrome patients including growth reduction, defects in communication, exploratory behavior as well as learning and memory, while social behavior and sensor motor gating functions were normal (Gao et al., 2014; Hori et al., 2015). In zebrafish, auts2 is highly expressed in the developing brain and Morpholino-mediated knockdown resulted in microcephaly, reduced lower jaws, swimming defects and a reduced response to tactile stimuli (Beunders et al., 2013; Oksenberg et al., 2013).

Currently, data analyzing the function of Fbrsl1 in animal model systems are limited. The expression of fbrsl 1 has been analyzed in zebrafish and these data show that it is mainly expressed in the developing brain, but also in the spinal cord, the cranial ganglia and the somites (Kondrychyn et al., 2017). In Xenopus, fbrsll is expressed throughout early developmental stages (Ufartes et al., 2020). At tailbud stages, it is expressed in the brain and craniofacial structures including the branchial arches and the cranial nerves (Ufartes et al., 2020). Morpholinomediated Fbrsl1 knockdown resulted in craniofacial defects and the embryos showed cartilage hypoplasia as well as a reduction in brain size on the injected side (Figures 1A-C). Furthermore, the cranial nerves (Figure 1D) and motor neurons displayed impaired neuronal migration (Ufartes et al., 2020). Thus, the first functional data on Fbrsl1 in Xenopus development indicate that FBRSL1 may share similar functions with AUTS2 in neural development, but may also have a unique role in craniofacial development, which is also consistent with the findings in patients affected by the respective disorders.

\section{AUTS2 HAS NUCLEAR AND CYTOPLASMIC FUNCTIONS WHICH MAY BE SHARED BY FBRSL1}

For AUTS2 a dual function, acting either in the cytoplasm or in the nucleus of developing neurons has been described (Hori et al., 2014). In the nucleus, AUTS2 was identified as a component of the Polycomb repressive complex PRC1 (Gao et al., 2012; Gao et al., 2014). Polycomb repressive complexes are multiprotein complexes acting as epigenetic regulators during development (Aranda et al., 2015; Chittock et al., 2017). Traditionally, they exert their function as transcriptional repressors (Simon and Kingston, 2013; Chittock et al., 2017; Kassis et al., 2017). The 

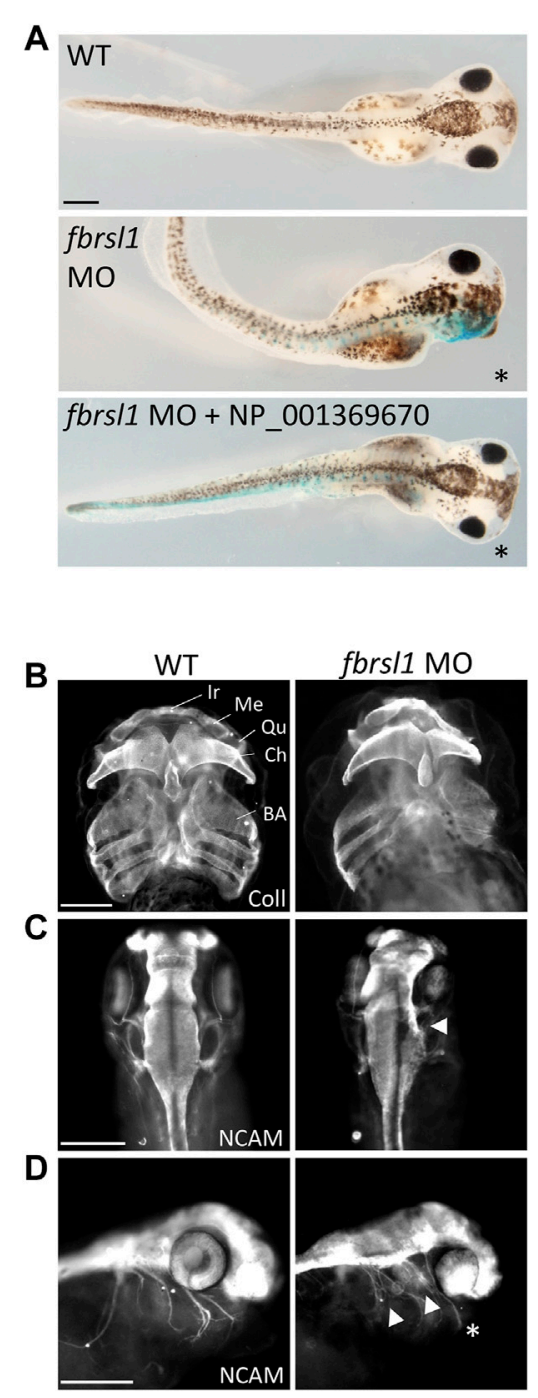

E

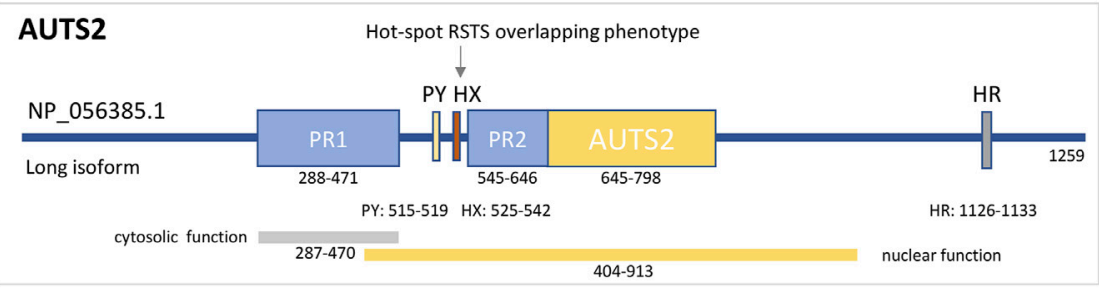

FBRSL1

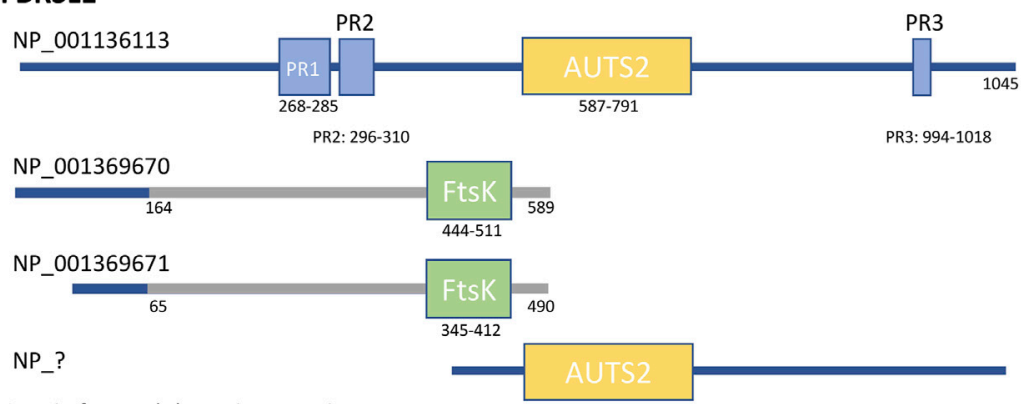

Long isoform and alternative transcripts

$\mathbf{F}$

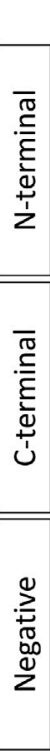

DAPI

FBRSL1

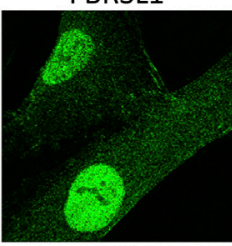

$\alpha$-TUBULIN
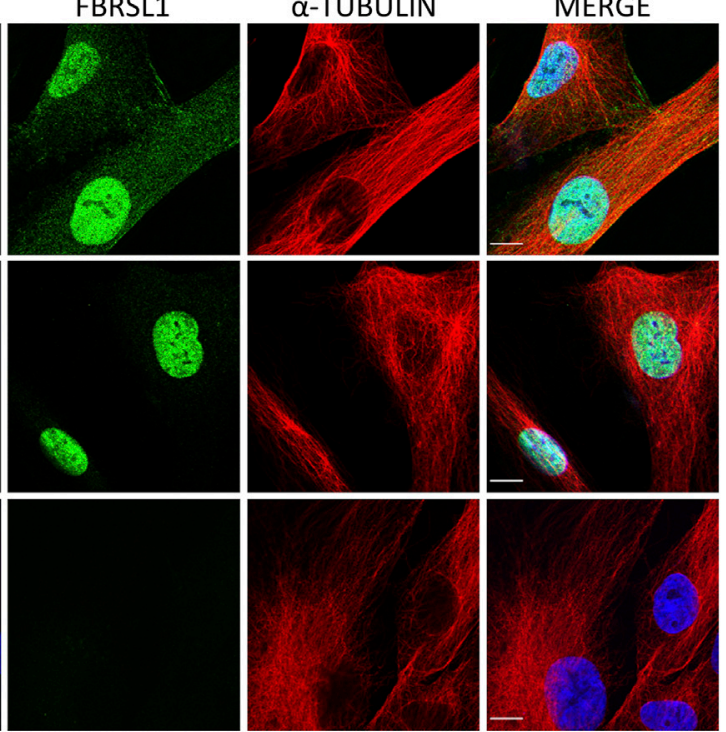

FIGURE 1 | Fbrsl1 knockdown phenotypes in Xenopus laevis and cellular localization of distinct human FBRSL1 transcripts. (A) Knockdown of Fbrsl1 by injection of a fbrs/1 Morpholino oligonucleotide (MO) leads to craniofacial defects that can be rescued by co-injection of RNA coding for the human short N-terminal FBRSL1 isoform NP_001369670 (Ufartes et al., 2020). LacZ RNA was co-injected as a lineage tracer, the injected side is marked by blue staining. (B) Anti-Collagen Type II immunofluorescence visualizes the cartilage and indicates cartilage hypoplasia on the fbrs/1 MO-injected side. BA, branchial arches; Ch, ceratohyal; Ir, infrarostral; Me, Meckel's cartilage; Qu, quadrate. (C) Neural cell adhesion molecule (NCAM) staining shows reduced brain size and (D) impaired outgrowth of cranial nerves on the fbrs/1 MO-injected side; * marks the injected side. Scale bar in A-D: 500 Mm. (E) Human FBRSL1 transcripts/isoforms compared to the human AUTS2 long isoform as previously published (Sultana et al., 2002; Oksenberg and Ahituv, 2013; Sellers et al., 2020). Like its AUTS2 ohnolog, the long isoform has a AUTS2 domain and proline rich (PR) regions (predicted with MobiDB (Piovesan et al., 2020)). Both short N-terminal isoforms differ in their C-terminal sequence from the long isoform (presented in grey) due to an alternative exon 3, which contains an Ftsk-domain. In addition, a predicted C-terminal isoform (marked with "?") including the AUTS2 domain is shown as this isoform was validated for mouse Fbrsl1. PR, proline-rich domain; PY, PPPY motif; HX, hexanucleotide repeat; HR, trinucleotide (H) repeat; AUTS2, AUTS2/FBRSL1/FBRSL homology region. (F) Immunofluorescence analysis performed on human fibroblasts. Antibodies directed against the $\mathrm{N}$-terminal as well as the C-terminal part of FBRSL1 detected FBRSL1 isoforms (green) in the nucleus. However, only the N-terminal FBRSL1 antibody also detected FBRSL1 in the cytoplasm, suggesting that the short N-terminal FBRSL1 isoforms show cytoplasmic and nuclear localization. The negative control showed no signal. Cytoskeletal staining was detected using an $\alpha$-Tubulin antibody and nuclei were stained using DAPI. Images were obtained using a confocal laser microscope with $\times 600$ magnification. Scale Bar: $10 \mu \mathrm{m}$. All experimental data have been previously published (Ufartes et al., 2020). 
two main Polycomb complexes are PRC1 and PRC2 (reviewed in Barbour et al., 2020; Cohen et al., 2020; Geng and Gao, 2020). The PRC1 complex acts as an E3 ubiquitin ligase that monoubiquitinates lysine 119 of histone H2A (H2AK119ub1) (De Napoles et al., 2004; Wang et al., 2004). The consequences of Polycomb dependent histone H2A ubiquitination were recently reviewed by Tamburri et al. (2021). In addition, the PRC1 complex is involved in ubiquitination-independent chromatin compaction (Eskeland et al., 2010). At least six PRC1 subcomplexes (PRC1.1-PRC1.6) were identified consisting of the E3 ubiquitin ligase RING1A or RING1B and one of the six Polycomb Group Ring Fingers (PCGF1-6) (Chittock et al., 2017; Varlet et al., 2020). AUTS2 has been described as a component of the subcomplexes PRC1.3 and PRC1.5 (Gao et al., 2012; Gao et al., 2014). The PRC1.5 complex contains the components AUTS2, PCGF5, RING1B, CK2B, and RYBP (Gao et al., 2012; Gao et al., 2014). The binding of AUTS2 to the PRC1.5 complex switches its function to a transcriptional activator by recruiting the histone acetyltransferase EP300 and casein kinase 2 (CK2) (Gao et al., 2014; Liu et al., 2021). Coimmunoprecipitation experiments revealed that RING1B interacts with AUTS2 only in the presence of PCGF5 (Gao et al., 2014), suggesting a bridging function of PCGF5. The recruitment of CK2 to the complex is likely mediated by direct AUTS2 binding and this interaction suppresses monoubiquitination of H2AK119 by RING1B (Gao et al., 2014). The C-terminal part of AUTS2 (404-913) is sufficient to mediate the transcriptional activation via EP300 binding (Gao et al., 2014). Therefore, the recruitment of CK2 to the PRC1.5 complex and the AUTS2-EP300 interaction seem to be responsible for converting the repressive PRC1 function into an activator function (Gao et al., 2014; MondererRothkoff et al., 2021). Recently, de novo pathogenic variants in the HX repeat region of AUTS2 were described in patients with a phenotype overlapping with Rubinstein-Taybi syndrome (Liu et al., 2021). Rubinstein-Taybi syndrome (RSTS, OMIM 180849 and OMIM 613684) is a neurodevelopmental disorder characterized by intellectual disability, autism spectrum disorders, microcephaly, facial dysmorphism, growth retardation, large thumbs and hallux and a variable degree of additional malformations and symptoms (reviewed in Van Gils et al., 2021). The underlying cause of RSTS are pathogenic variants in EP300 and CREBBP (Petrij et al., 1995; Roelfsema et al., 2005). Interestingly, the AUTS2 variants leading to an RSTS-overlapping phenotype disrupt the binding of AUTS2 to EP300, suggesting that the HX repeat domain is responsible for this interaction (Liu et al., 2021). The binding of AUTS2 to PRC1.3 and the recruitment to chromatin was shown to be directed by the transcription factor nuclear respiratory factor 1 (NRF1). In motor neurons, AUTS2 and NRF1 colocalize at actively transcribed loci, whereby AUTS2 binding requires NRF1, but NRF1 binding is independent of AUTS2 (Liu et al., 2021). Thus, it was suggested that NRF1 recruits AUTS2 in the context of the PRC1.3 complex to genes involved in neuronal differentiation. The transcription of these genes will then be activated by binding of EP300 to the AUTS2 HX repeat domain (Liu et al., 2021).
Like AUTS2, FBRSL1 was also identified by tandem affinity purification and mass spectrometry as an interaction partner of PRC1.3 and PRC1.5 (Gao et al., 2012). Further, it was shown that FBRSL1 competes with AUTS2 for binding to the PRC1.5 complex (Gao et al., 2014). Thus, it will be interesting to see if a PRC1.3 or PRC1.5 complex containing FBRSL1 in place of AUTS2 has again a repressive function instead of an active role. While interaction of FBRSL1 and AUTS2 with Polycomb complexes indicates a role of these proteins in transcriptional gene regulation, they likely also control additional processes in the cytoplasm.

For AUTS2 it has been shown that-in addition to its function in the nucleus-it also functions in the cytoplasm by controlling cytoskeletal dynamics. Cytoplasmic AUTS2 functions by regulating small GTPases of the Rho family thereby affecting actin dynamics in the developing brain (Hori et al., 2014). By stimulating small guanine exchange factors (GEFs) AUTS2 activates Rac1 and induces lamellipodia formation and neurite extension. Conversely, AUTS2 inhibits Cdc42 thereby suppressing filopodia formation (Hori et al., 2014). For Rac1 activation, the N-terminal PR1 region of the AUTS2 protein seems to be important, as overexpression of mutant AUTS2, lacking the N-terminal PR1 domain, did not lead to lamellipodia formation (Hori et al., 2014). Currently, it is unknown if FBRSL1 may play a similar role. However, we recently demonstrated that FBRSL1 is localized in the cytoplasm as well as in the nucleus of HEK293 cells and human fibroblasts (Figures 1E,F) (Ufartes et al., 2020). Consistent with the AUTS2 data (Hori et al., 2014), mainly a nuclear FBRSL1 pattern was detected with an antibody directed against the C-terminal part of FBRSL1, while nuclear and cytoplasmic FBRSL1 was observed with an antibody targeted against the N-terminal part of the protein (Figure 1F) (Ufartes et al., 2020). Thus, it is likely that FBRSL1-like AUTS2-has nuclear versus cytoplasmic functions which may require distinct domains of the protein.

\section{FBRSL1 AND AUTS2 SHOW TRANSCRIPTIONAL COMPLEXITY}

Consistent with the concept of distinct subcellular functions, different transcripts have been identified for AUTS2 and FBRSL1. The longest AUTS2 and FBRSL1 transcripts are encoded by 19 exons, in addition shorter $\mathrm{N}$-terminal or $\mathrm{C}$-terminal transcripts of the respective proteins have been described. For AUTS2, two isoforms have been extensively studied: the long transcript containing 19 exons (NM_015570.4) and a short C-terminal isoform containing the last 11 exons, starting at exon 9, first characterized by Beunders et al., 2013 (Beunders et al., 2013). The C-terminal isoform contains a region of homology to the paralogs FBRSL1 and FBRS, which was called AUTS2 family domain (Kondrychyn et al., 2017), and is critical for the nuclear function of AUTS2 (Beunders et al., 2013). Beunders et al. showed that the characteristic dysmorphic features were more pronounced in patients with 3' AUTS2 deletions (Beunders et al., 2013). Furthermore, they showed that Morpholino-mediated knockdown of zebrafish Auts2 resulted in microcephaly and 
reduced lower jaw size, comparable to defects seen in patients with an AUTS2 disruption. The morphant phenotypes could be rescued with wild-type human full-length Auts2 RNA, but also with a short C-terminal Auts2 isoform encoded by exons 9-19 (Beunders et al., 2013) demonstrating the important role for the AUTS2 C-terminus during development.

Like for AUTS2, a long FBRSL1 transcript containing 19 exons (NM_001142641.2) was identified (Figure 1E) (Ufartes et al., 2020). In addition, two N-terminal isoforms were validated (Figure 1E) and studied in more detail. The two short isoforms contain an alternative exon three leading to a stop codon. These two short N-terminal forms lack the homologous AUTS2 family domain, but include a predicted DNA translocase domain (Ftsk) (NCBI conserved database, CDD) (Ufartes et al., 2020). Interestingly, while the severe AUTS2 syndrome phenotype was caused by variants of the C-terminus (Beunders et al., 2013), the situation was different for the three patients with the FBRSL1associated syndromic phenotype: all three patients harbor a truncating variant (stop mutation in two patients and a frameshift variant with premature stop codon in the other patient) in the N-terminus of FBRSL1 affecting the short N-terminal isoforms (Ufartes et al., 2020). Consistently, using the Xenopus systems, we could show that a human N-terminal isoform of FBRSL1 was able to rescue the Xenopus morphant craniofacial defects. However, neither a patient variant of this isoform nor the long FBRSL1 isoform, which both lack the Ftsk domain, were able to rescue the morphant phenotype (Ufartes et al., 2020). These data suggest that mutations of the short N-terminal FBRSL1 isoforms are causative for the developmental phenotype in the animal model system and possibly also in human patients.

It is tempting to speculate that this transcriptional complexity is also responsible for the distinct functions of FBRSL1 and AUTS2. For example, in zebrafish it has been shown that the transcriptional complexity of distinct Auts2 family ohnologs is mediated by alternative splicing and alternative promotor use (Kondrychyn et al., 2017). Interestingly, the expression of the distinct Auts2 paralogs is temporally and spatially tightly controlled during development (Kondrychyn et al., 2017). Thus, there are multiple levels, by which distinct functions can be regulated by this gene family.

\section{REFERENCES}

Aranda, S., Mas, G., and Di Croce, L. (2015). Regulation of Gene Transcription by Polycomb Proteins. Sci. Adv. 1, e1500737. doi:10.1126/sciadv.1500737

Bakkaloglu, B., O’roak, B. J., Louvi, A., Gupta, A. R., Abelson, J. F., Morgan, T. M., et al. (2008). Molecular Cytogenetic Analysis and Resequencing of Contactin Associated Protein-like 2 in Autism Spectrum Disorders. Am. J. Hum. Genet. 82, 165-173. doi:10.1016/j.ajhg.2007.09.017

Barbour, H., Daou, S., Hendzel, M., and Affar, E. B. (2020). Polycomb Group-Mediated Histone H2A Monoubiquitination in Epigenome Regulation and Nuclear Processes. Nat. Commun. 11, 5947. doi:10.1038/s41467-020-19722-9

Bedogni, F., Hodge, R. D., Nelson, B. R., Frederick, E. A., Shiba, N., Daza, R. A., et al. (2010). Autism Susceptibility Candidate 2 (Auts2) Encodes a Nuclear Protein Expressed in Developing Brain Regions Implicated in Autism Neuropathology. Gene Expr. Patterns 10, 9-15. doi:10.1016/j.gep.2009.11.005

\section{CONCLUSION}

According to currently available data on FBRSL1, we would speculate that the N-terminal region of FBRSL1, has an important function in mammalian development. This hypothesis is also supported by the finding that all three patients, affected by a novel severe malformation syndrome, carry FBRSL1 variants localizing to the $\mathrm{N}$-terminal region of FBRSL1. Although these patients show overlapping features to patients with AUTS2 syndrome, which is caused by variants in the FBRSL1-paralog AUTS2, they have a higher rate and wider spectrum of congenital malformations. As the number of described patients with FBRSL1 variants are currently small, larger patient cohorts with clinical description of the disease are required to confirm these first observations. FBRSL1 and AUTS2 are closely related paralogs, but the presently published data indicate that they have distinct functions and cannot replace each other. Thus, future research will need to address the molecular and cellular mechanism of FBRSL1 to reveal its unique role in development and disease.

\section{AUTHOR CONTRIBUTIONS}

$\mathrm{AB}$ and SP conceptualized the article and wrote the original draft. $\mathrm{HB}$ and RU conceptualized and designed Figure $\mathbf{1}$ and critical revised the original draft.

\section{FUNDING}

This work was supported by the Deutsche Forschungsgemeinschaft (DFG, German Research Foundation) grant PA 2030/5-1 to SP.

\section{ACKNOWLEDGMENTS}

The authors thank Katharina Till for critical reading of the manuscript.

Beunders, G., Van De Kamp, J., Vasudevan, P., Morton, J., Smets, K., Kleefstra, T., et al. (2016). A Detailed Clinical Analysis of 13 Patients with AUTS2 Syndrome Further Delineates the Phenotypic Spectrum and Underscores the Behavioural Phenotype. J. Med. Genet. 53, 523-532. doi:10.1136/jmedgenet-2015-103601

Beunders, G., Voorhoeve, E., Golzio, C., Pardo, L. M., Rosenfeld, J. A., Talkowski, M. E., et al. (2013). Exonic Deletions in AUTS2 Cause a Syndromic Form of Intellectual Disability and Suggest a Critical Role for the C Terminus. Am. J. Hum. Genet. 92, 210-220. doi:10.1016/j.ajhg.2012.12.011

Chittock, E. C., Latwiel, S., Miller, T. C. R., and Müller, C. W. (2017). Molecular Architecture of Polycomb Repressive Complexes. Biochem. Soc. Trans. 45, 193-205. doi:10.1042/bst20160173

Cohen, I., Bar, C., and Ezhkova, E. (2020). Activity of PRC1 and Histone H2AK119 Monoubiquitination: Revising Popular Misconceptions. Bioessays 42, e1900192. doi:10.1002/bies.201900192

De Napoles, M., Mermoud, J. E., Wakao, R., Tang, Y. A., Endoh, M. Appanah, R., et al. (2004). Polycomb Group Proteins Ring1A/B Link 
Ubiquitylation of Histone $\mathrm{H} 2 \mathrm{~A}$ to Heritable Gene Silencing and X Inactivation. Developmental $\mathrm{Cel} 7,663-676$ doi:10.1016/ j.devcel.2004.10.005

Dickerson, J. E., and Robertson, D. L. (2012). On the Origins of Mendelian Disease Genes in Man: the Impact of Gene Duplication. Mol. Biol. Evol. 29, 61-69. doi:10.1093/molbev/msr111

Eskeland, R., Leeb, M., Grimes, G. R., Kress, C., Boyle, S., Sproul, D., et al. (2010). Ring1B Compacts Chromatin Structure and Represses Gene Expression Independent of Histone Ubiquitination. Mol. Cel 38, 452-464. doi:10.1016/ j.molcel.2010.02.032

Gao, Z., Lee, P., Stafford, J. M., Von Schimmelmann, M., Schaefer, A., and Reinberg, D. (2014). An AUTS2-Polycomb Complex Activates Gene Expression in the CNS. Nature 516, 349-354. doi:10.1038/nature13921

Gao, Z., Zhang, J., Bonasio, R., Strino, F., Sawai, A., Parisi, F., et al. (2012). PCGF Homologs, CBX Proteins, and RYBP Define Functionally Distinct PRC1 Family Complexes. Mol. Cel 45, 344-356. doi:10.1016/ j.molcel.2012.01.002

Geng, Z., and Gao, Z. (2020). Mammalian PRC1 Complexes: Compositional Complexity and Diverse Molecular Mechanisms. Int. J. Mol. Sci. 21. doi:10.3390/ijms21228594

Girirajan, S., Brkanac, Z., Coe, B. P., Baker, C., Vives, L., Vu, T. H., et al. (2011). Relative burden of Large CNVs on a Range of Neurodevelopmental Phenotypes. Plos Genet. 7, e1002334. doi:10.1371/journal.pgen.1002334

Hori, K., and Hoshino, M. (2017). Neuronal Migration and AUTS2 Syndrome. Brain Sci. 7. doi:10.3390/brainsci7050054

Hori, K., Nagai, T., Shan, W., Sakamoto, A., Abe, M., Yamazaki, M., et al. (2015). Heterozygous Disruption of Autism Susceptibility Candidate 2 Causes Impaired Emotional Control and Cognitive Memory. PLoS One 10, e0145979. doi:10.1371/journal.pone.0145979

Hori, K., Nagai, T., Shan, W., Sakamoto, A., Taya, S., Hashimoto, R., et al. (2014). Cytoskeletal Regulation by AUTS2 in Neuronal Migration and Neuritogenesis. Cel Rep. 9, 2166-2179. doi:10.1016/j.celrep.2014.11.045

Huang, X.-L., Zou, Y. S., Maher, T. A., Newton, S., and Milunsky, J. M. (2010). A De Novo Balanced Translocation Breakpoint Truncating the Autism Susceptibility Candidate 2 (AUTS2) Gene in a Patient with Autism. Am. J. Med. Genet. 152A, 2112-2114. doi:10.1002/ajmg.a.33497

Jolley, A., Corbett, M., Mcgregor, L., Waters, W., Brown, S., Nicholl, J., et al. (2013). De Novo intragenic Deletion of Theautism Susceptibility Candidate 2(AUTS2) Gene in a Patient with Developmental Delay: A Case Report and Literature Review. Am. J. Med. Genet. 161, 1508-1512. doi:10.1002/ajmg.a.35922

Kalscheuer, V. M., Fitzpatrick, D., Tommerup, N., Bugge, M., Niebuhr, E., Neumann, L. M., et al. (2007). Mutations in Autism Susceptibility Candidate 2 (AUTS2) in Patients with Mental Retardation. Hum. Genet. 121, 501-509. doi:10.1007/s00439-006-0284-0

Kassis, J. A., Kennison, J. A., and Tamkun, J. W. (2017). Polycomb and Trithorax Group Genes in Drosophila. Genetics 206, 1699-1725. doi:10.1534/ genetics.115.185116

Kondrychyn, I., Robra, L., and Thirumalai, V. (2017). Transcriptional Complexity and Distinct Expression Patterns of Auts2 Paralogs in Danio rerio. G3 (Bethesda) 7, 2577-2593. doi:10.1534/g3.117.042622

Liu, S., Aldinger, K. A., Cheng, C. V., Kiyama, T., Dave, M., Mcnamara, H. K., et al. (2021). NRF1 Association with AUTS2-Polycomb Mediates Specific Gene Activation in the Brain. Mol. Cel S1097-2765, 00754-761. doi:10.1016/ j.molcel.2021.09.020

Liu, Y., Zhao, D., Dong, R., Yang, X., Zhang, Y., Tammimies, K., et al. (2015). De Novo exon 1 Deletion ofAUTS2gene in a Patient with Autism Spectrum Disorder and Developmental Delay: A Case Report and a Brief Literature Review. Am. J. Med. Genet. 167, 1381-1385. doi:10.1002/ajmg.a.37050

Malaguti, G., Singh, P. P., and Isambert, H. (2014). On the Retention of Gene Duplicates Prone to Dominant Deleterious Mutations. Theor. Popul. Biol. 93, 38-51. doi:10.1016/j.tpb.2014.01.004

Mclysaght, A., Makino, T., Grayton, H. M., Tropeano, M., Mitchell, K. J., Vassos, E., et al. (2014). Ohnologs Are Overrepresented in Pathogenic Copy Number Mutations. Proc. Natl. Acad. Sci. USA 111, 361-366. doi:10.1073/ pnas. 1309324111
Monderer-Rothkoff, G., Tal, N., Risman, M., Shani, O., Nissim-Rafinia, M., MalkiFeldman, L., et al. (2021). AUTS2 Isoforms Control Neuronal Differentiation. Mol. Psychiatry 26, 666-681. doi:10.1038/s41380-019-0409-1

Nagamani, S. C. S., Erez, A., Ben-Zeev, B., Frydman, M., Winter, S., Zeller, R., et al. (2013). Detection of Copy-Number Variation in AUTS2 Gene by Targeted Exonic Array CGH in Patients with Developmental Delay and Autistic Spectrum Disorders. Eur. J. Hum. Genet. 21, 343-346. doi:10.1038/ ejhg.2012.157

Oksenberg, N., and Ahituv, N. (2013). The Role of AUTS2 in Neurodevelopment and Human Evolution. Trends Genet. 29, 600-608. doi:10.1016/ j.tig.2013.08.001

Oksenberg, N., Stevison, L., Wall, J. D., and Ahituv, N. (2013). Function and Regulation of AUTS2, a Gene Implicated in Autism and Human Evolution. Plos Genet. 9, e1003221. doi:10.1371/journal.pgen.1003221

Pang, W., Yi, X., Li, L., Liu, L., Xiang, W., and Xiao, L. (2021). Untangle the Multi-Facet Functions of Auts2 as an Entry Point to Understand Neurodevelopmental Disorders. Front. Psychiatry 12, 580433. doi:10.3389/fpsyt.2021.580433

Petrij, F., Giles, R. H., Dauwerse, H. G., Saris, J. J., Hennekam, R. C., Masuno, M., et al. (1995). Rubinstein-Taybi Syndrome Caused by Mutations in the Transcriptional Co-activator CBP. Nature 376, 348-351. doi:10.1038/ 376348a0

Piovesan, D., Hatos, A., Minervini, G., Quaglia, F., Monzon, A. M., and Tosatto, S. C. E. (2020). Assessing Predictors for New post Translational Modification Sites: A Case Study on Hydroxylation. Plos Comput. Biol. 16, e1007967. doi:10.1371/journal.pcbi.1007967

Roelfsema, J. H., White, S. J., Ariyürek, Y., Bartholdi, D., Niedrist, D., Papadia, F., et al. (2005). Genetic Heterogeneity in Rubinstein-Taybi Syndrome: Mutations in Both the CBP and EP300 Genes Cause Disease. Am. J. Hum. Genet. 76, 572-580. doi:10.1086/429130

Saeki, S., Enokizono, T., Imagawa, K., Fukushima, H., Kajikawa, D., Sakai, A., et al. (2019). A Case of Autism Spectrum Disorder with Cleft Lip and Palate Carrying a Mutation in Exon 8 of AUTS2. Clin. Case Rep. 7, 2059-2063. doi:10.1002/ ccr3.2377

Sanchez-Jimeno, C., Blanco-Kelly, F., López-Grondona, F., Losada-Del Pozo, R., Moreno, B., Rodrigo-Moreno, M., et al. (2021). Attention Deficit Hyperactivity and Autism Spectrum Disorders as the Core Symptoms of AUTS2 Syndrome: Description of Five New Patients and Update of the Frequency of Manifestations and Genotype-Phenotype Correlation. Genes 12, 1360. doi:10.3390/genes12091360

Sellers, R. A., Robertson, D. L., and Tassabehji, M. (2020). Ancestry of the AUTS2 Family-A Novel Group of Polycomb-Complex Proteins Involved in Human Neurological Disease. PLoS One 15, e0232101. doi:10.1371/ journal.pone.0232101

Simon, J. A., and Kingston, R. E. (2013). Occupying Chromatin: Polycomb Mechanisms for Getting to Genomic Targets, Stopping Transcriptional Traffic, and Staying Put. Mol. Cel 49, 808-824. doi:10.1016/ j.molcel.2013.02.013

Singh, P. P., Affeldt, S., Cascone, I., Selimoglu, R., Camonis, J., and Isambert, H. (2012). On the Expansion of "dangerous" Gene Repertoires by Whole-Genome Duplications in Early Vertebrates. Cel Rep. 2, 1387-1398. doi:10.1016/ j.celrep.2012.09.034

Singh, P. P., Arora, J., and Isambert, H. (2015). Identification of Ohnolog Genes Originating from Whole Genome Duplication in Early Vertebrates, Based on Synteny Comparison across Multiple Genomes. Plos Comput. Biol. 11, e1004394. doi:10.1371/journal.pcbi.1004394

Sultana, R., Yu, C.-E., Yu, J., Munson, J., Chen, D., Hua, W., et al. (2002). Identification of a Novel Gene on Chromosome 7q11.2 Interrupted by a Translocation Breakpoint in a Pair of Autistic Twins. Genomics 80, 129-134. doi:10.1006/geno.2002.6810

Tamburri, S., Conway, E., and Pasini, D. (2021). Polycomb-dependent Histone H2A Ubiquitination Links Developmental Disorders with Cancer. Trends Genet. 11. doi:10.1016/j.tig.2021.07.011

Ufartes, R., Berger, H., Till, K., Salinas, G., Sturm, M., Altmuller, J., et al. (2020). De Novo mutations in FBRSL1 Cause a Novel Recognizable Malformation and Intellectual Disability Syndrome. Hum. Genet. 139, 1363-1379. doi:10.1007/ s00439-020-02175-x 
Van Gils, J., Magdinier, F., Fergelot, P., and Lacombe, D. (2021). Rubinstein-Taybi Syndrome: A Model of Epigenetic Disorder. Genes (Basel) 12. doi:10.3390/ genes 12070968

Varlet, E., Ovejero, S., Martinez, A. M., Cavalli, G., and Moreaux, J. (2020). Role of Polycomb Complexes in Normal and Malignant Plasma Cells. Int. J. Mol. Sci. 21. doi:10.3390/ijms 21218047

Wang, H., Wang, L., Erdjument-Bromage, H., Vidal, M., Tempst, P., Jones, R. S., et al. (2004). Role of Histone H2A Ubiquitination in Polycomb Silencing. Nature 431, 873-878. doi:10.1038/nature02985

Conflict of Interest: The authors declare that the research was conducted in the absence of any commercial or financial relationships that could be construed as a potential conflict of interest.
Publisher's Note: All claims expressed in this article are solely those of the authors and do not necessarily represent those of their affiliated organizations, or those of the publisher, the editors and the reviewers. Any product that may be evaluated in this article, or claim that may be made by its manufacturer, is not guaranteed or endorsed by the publisher.

Copyright $\odot 2021$ Pauli, Berger, Ufartes and Borchers. This is an open-access article distributed under the terms of the Creative Commons Attribution License (CC BY).

The use, distribution or reproduction in other forums is permitted, provided the original author(s) and the copyright owner(s) are credited and that the original publication in this journal is cited, in accordance with accepted academic practice. No use, distribution or reproduction is permitted which does not comply with these terms. 